\title{
A PROPERTY OF IDEALS IN POLYNOMIAL RINGS
}

\author{
GENNADY LYUBEZNIK
}

\begin{abstract}
Every ideal in the polynomial ring in $n$ variables over an infinite field has a reduction generated by $n$ elements.
\end{abstract}

Eisenbud and Evans [2] proved that every ideal in $k\left[X_{1}, \ldots, X_{n}\right]$ can be generated up to radical by $n$ elements (where $k$ is a field). Avinash Sathaye [7] and Mohan Kumar [5] proved a locally complete intersection in $k\left[X_{1}, \ldots, X_{n}\right]$ can be generated by $n$ elements.

In this short note we show that every ideal in $k\left[X_{1}, \ldots, X_{n}\right]$ has a nice approximation generated by $n$ elements. More precisely, we prove the following.

THEOREM. Let $k$ be an infinite field. Then every ideal $I$ in $k\left[X_{1}, \ldots, X_{n}\right]$ has a reduction $J$ generated by $n$ elements.

By [6], $J$ is a reduction of $I$ if there exists an integer $r$ such that $J I^{r}=I^{r+1}$. Northcott and Rees [6] point out that $J$ can be regarded as a simplified version of $I$ preserving many properties of $I$, in particular the multiplicities at minimal prime over-ideals. Moreover, $J$ has the same radical as $I$ and if $I$ is locally a complete intersection, then it is the only reduction of itself, hence a connection between our result and those of Eisenbud and Evans and Sathaye and Kumar.

Proof OF THE THEOREM. Since $k\left[X_{1}, \ldots, X_{n}\right]$ is a UFD, we can assume that $n \geqslant \operatorname{dim}(A / I)+2$. Let $g_{1}, \ldots, g_{r}$ be a system of generators of $I$. Set $A=$ $k\left[X_{1}, \ldots, X_{n}\right]$ and $B=k\left[g_{1}, \ldots, g_{r}\right] \subset A$. The dimension of $B$ is at most $n$, since its quotient field is a subfield of $k\left(X_{1}, \ldots, X_{n}\right)$ and therefore has transcendence degree $\leqslant n$. Denote by $P$ the ideal of $B$ generated by $g_{1}, \ldots, g_{r}$. Let $\bar{P}$ be the image of $P$ in $B_{P}$, the localization of $B$ at $P$ (i.e. $\bar{P}$ is the maximal ideal of $B_{P}$ ). Since $B_{P}$ is local of dimension $\leqslant n$, by Burch [1] $\bar{P}$ has a reduction $\bar{Q}$ generated by $n$ elements $h_{1}, \ldots, h_{n}$. Let $r$ be an integer such that $\overline{Q P}^{r}=\bar{P}^{r+1}$. Since $\bar{Q}$ is $\bar{P}$-primary, there exists a unique $P$-primary ideal $Q \subset B$, the image of which in $B_{P}$ coincides with $\bar{Q}$. Since $P$ is a maximal ideal of $B$, we see that the ideals $Q P^{r}$ and $P^{r+1}$ are $P$-primary and their images in $B_{P}$ coincide. Therefore, $Q P^{r}=P^{r+1}$ in $B$, since there is a one-to-one correspondence between the $\bar{P}$-primary ideals of $B_{P}$ and the $P$-primary ideals of $B$.

Let $J$ be the extension of $Q$ in $A$. Since the extension of $P$ is $I$ and $Q P^{r}=P^{r+1}$, we see that $J I^{r}=I^{r+1}$, i.e. $J$ is a reduction of $I$.

Received by the editors September 13, 1985.

1980 Mathematics Subject Classification (1985 Revision). Primary 13F20, 13 A15.

(C)1986 American Mathematical Society $0002-9939 / 86 \$ 1.00+\$ .25$ per page 
Let $s \in B \backslash P$ be an element such that $Q_{s}=\left(h_{1}, \ldots, h_{n}\right)$. Thus $J_{s}=\left(h_{1}, \ldots, h_{n}\right)$. Since $Q$ is $P$-primary and $P$ is a maximal ideal of $B$, we see that $(s)+Q=(1)$, i.e., regarding $s$ as an element of $A$, that $(s)+J=(1)$. The conditions $(s)+J=(1)$ and $J_{s}=\left(h_{1}, \ldots, h_{n}\right)$ imply that $J / J^{2}$ is generated by $n$ elements $h_{1}, \ldots, h_{n}$. Now Theorem 5 of Mohan Kumar [4] tells us that $J$ is generated by $n$ elements, since $n \geqslant \operatorname{dim}(A / J)+2$. Q.E.D.

REMARKS. 1. In a similar way one can prove that every ideal in a finitely generated $n$-dimensional algebra over an infinite field has a reduction generated by $n+1$ elements.

2. For every prime ideal $P \subset B$ containing $I$ we have the dimension of

$$
(J+P \cdot I) / P \cdot I
$$

as a vector space over the quotient field of $A / P$ is $\geqslant$ height $I$. This improves Eisenbud and Evans [2,3] who proved only that $J \not \subset P \cdot I$.

3. Some generalization of our theorem is possible to the case $I \subset A[X]$, where $A$ is an $(n-1)$-dimensional finitely generated algebra over an infinite field. For this one has to use S. Mandall's extension of Mohan Kumar's theorem to ideals in $A[X]$ (cf. [5]).

CONJECTURE. Let $A$ be a commutative Noetherian ring of dimension $n-1$ such that the residue field of every maximal ideal of $A$ is infinite. Let $I$ be an ideal of $A$ or $A[X]$. Then $I$ has a reduction generated by $n$ elements.

\section{REFERENCES}

1. L. Burch, Codimension and analytic spread, Proc. Cambridge Philos. Soc. 72 (1972), 369-373.

2. D. Eisenbud and E. Evans, Every algebraic set in $n$-space is a set-theoretic intersection of $n$ hypersurfaces, Invent. Math. 19 (1973), 107-112.

3. , Generating modules efficiently: Theorems from algebraic K-theory, J. Algebra 27 (1973), 278-305.

4. Mohan Kumar, On two conjectures about polynomial rings, Invent. Math. 46 (1978), 225-236.

5. S. Mandall, On efficient generation of ideals, Invent. Math. 75 (1984), 59-67.

6. D. G. Northcott and D. Rees, Reductions of ideals in local rings, Proc. Cambridge Philos. Soc. 50 (1954), 147-158.

7. A. Sathaye, On the Forster-Eisenbud-Evans conjectures, Invent. Math. 46 (1978), 211-224.

Department of Mathematics, PURdue University, West Lafa yette, Indiana 47907

Current address: Department of Mathematics, The University of Chicago, 5734 University Avenue, Chicago, Illinois 60637 